\title{
The complete mitochondrial genome of the hermaphroditic freshwater mussel Anodonta cygnea (Bivalvia: Unionidae): in silico analyses of sex-specific ORFs across order Unionoida
}

\author{
E. E. Chase ${ }^{1}$, B. M. Robicheau ${ }^{2}$, S. Veinot ${ }^{2}$, S. Breton ${ }^{3}$ and D. T. Stewart ${ }^{1 *}$ (i)
}

\begin{abstract}
Background: Doubly uniparental inheritance (DUI) of mitochondrial DNA in bivalves is a fascinating exception to strictly maternal inheritance as practiced by all other animals. Recent work on DUI suggests that there may be unique regions of the mitochondrial genomes that play a role in sex determination and/or sexual development in freshwater mussels (order Unionoida). In this study, one complete mitochondrial genome of the hermaphroditic swan mussel, Anodonta cygnea, is sequenced and compared to the complete mitochondrial genome of the gonochoric duck mussel, Anodonta anatina. An in silico assessment of novel proteins found within freshwater bivalve species (known as F-, $\mathrm{H}$-, and M-open reading frames or ORFs) is conducted, with special attention to putative transmembrane domains (TMs), signal peptides (SPs), signal cleavage sites (SCS), subcellular localization, and potential control regions. Characteristics of TMs are also examined across freshwater mussel lineages.
\end{abstract}

Results: In silico analyses suggests the presence of SPs and SCSs and provides some insight into possible function(s) of these novel ORFs. The assessed confidence in these structures and functions was highly variable, possibly due to the novelty of these proteins. The number and topology of putative TMs appear to be maintained among both F- and H-ORFs, however, this is not the case for M-ORFs. There does not appear to be a typical control region in $\mathrm{H}$-type mitochondrial DNA, especially given the loss of tandem repeats in unassigned regions when compared to F-type mtDNA.

Conclusion: In silico analyses provides a useful tool to discover patterns in DUI and to navigate further in situ analyses related to DUI in freshwater mussels. In situ analysis will be necessary to further explore the intracellular localizations and possible role of these open reading frames in the process of sex determination in freshwater mussel.

Keywords: Doubly uniparental inheritance, Signal peptide, Transmembrane domain, Subcellular localization, Mitochondria, Hermaphroditism

\footnotetext{
* Correspondence: don.stewart@acadiau.ca

${ }^{1}$ Department of Biology, Acadia University, Wolfville, NS, Canada

Full list of author information is available at the end of the article
} 


\section{Background}

The order Unionoida is composed of freshwater mussels (FWM) that inhabit rivers, lakes and ponds [1]. Many gonochoric FWMs (i.e. species with separate male and female sexes), along with a few other bivalve orders (i.e. Mytiloid marine mussels, Nuculanoid nut shells, and Veneroid marine clams), do not exhibit strictly maternal inheritance of mitochondrial DNA (mtDNA) like all other animal species [2]. In contrast, these bivalves transmit mtDNA via an unusual system called doubly uniparental inheritance or DUI (for reviews see [3-5]). Under DUI, female offspring inherit maternal mtDNA (called female-transmitted or F-type mtDNA), while male offspring inherit both maternal mtDNA and paternal mtDNA (called male-transmitted or M-type mtDNA). Consequently, with respect to mitotype, males are heteroplasmic and females are homoplasmic [6]. In male offspring, F-type mtDNA is dominant throughout their somatic tissue, whereas M-type mtDNA is exclusive within gametic cells [7-9]. The respective M-type and F-type mtDNAs are also unusual, in contrast to typical animal mtDNA gene content, as each of the sexassociated mitochondrial genomes contains their own novel open reading frames (ORFs; [10]). These genes are referred to as the $m$-orf and $f$-orf in the male and female mitochondrial genomes, respectively, and these two regions code for proteins that do not show significant similarity to each other or obvious homology to any other known proteins ([10]; but also see [11, 12] who suggested that a duplicated and diverged atp 8 gene evolved into the m-orf gene in freshwater mussels). Immunostaining previously localized the F-ORF protein in not only the mitochondria of mature egg cells, but also on the nuclear membrane and in the nucleoplasm of the FWM Venustaconcha ellipsiformis (Bivalvia: Unionidae; [13]). The movement of the F-ORF protein from the mitochondria to the nucleus infers a function outside of the primary mitochondrial function of oxidative phosphorylation [13]. This finding, along with the transition of sexual strategies (detailed below) accompanied by a loss of the m-orf, suggests a link between sex determination and these novel ORFs in species exhibiting DUI [13]. The in silico analyses presented here continues the exploration of this potential link through locating putative transmembrane domains (TMs), signal peptides (SPs) and signal cleavage sites (SCSs). These molecular structures or signals may be key components of the pathway and/or mechanisms used to export these novel mitochondrial proteins to the nucleus where they could play a role in sex determination.

Recent work has shown that hermaphroditic FWMs do not possess M-type mtDNA [6, 13]. These hermaphroditic species have reverted to uniparental inheritance and transmit only an F-like mitochondrial genome, referred to as the hermaphroditic or $\mathrm{H}$-type genome [13]. In several hermaphroditic species, the H-type mtDNA carries a highly modified $f$-orf gene termed the $h$-orf [13]. Because hermaphroditism has evolved independently multiple times in FWMs, h-orfs from different genera are not monophyletic $[6,13]$. Breton et al. [13] identified four independent transitions from a gonochoric to hermaphroditic sexual strategy in the genera Lasmigona, Margaritifera, Toxolasma and Utterbackia. Interspecific comparisons of hermaphroditic FWM species showed that these $h$-orfs have highly divergent nucleotide sequences and variable amino acid hydrophobicity profiles, whereas f-orfs are more conserved in both respects $[6,13]$. Sequence analysis of the $f$-orfs and closely related $h$-orfs suggests that the molecular modifications of $h$-orfs typically include: (1) a lengthening of the sequence and (2) the introduction of repeating sequence motifs of varying lengths [13]. Based on these characteristics, it has been hypothesized that the $f$-orf and m-orf genes could be key elements of the sex determination pathway in gonochoric freshwater mussels with DUI, and that associated with the shift to hermaphroditism, the $h$-orf experiences relaxed purifying selection and a change (or loss) of function compared to the $f$-orf [13].

Given the unique nature of DUI, there is considerable interest in understanding the structure and function of the various elements present in bivalve $\mathrm{F}-, \mathrm{M}$ - and $\mathrm{H}$ type mitochondrial genomes. This includes characterizing the orfs and various unassigned regions (URs), and identifying putative control regions [4, 14, 15]. Such studies are important for a better understanding of sexual strategy transitions among FWMs and to gain insights into the possible functions of the novel proteincoding genes in bivalves with DUI. In this study, we present the complete mtDNA sequence of a fifth, independently evolved hermaphroditic FWM lineage in the genus Anodonta sampled from Lake Konstanz, Germany. One objective is to characterize potential control regions and URs of this A. cygnea H-type mitochondrial genome. It has been previously documented that the F- and M-type mtDNAs of FWMs with DUI share three sizable regions of non-coding DNA including the putative nad5-trnQ control region, as well as two areas flanking nad3 termed trnF-nad3 and nad3-trnH $+A$ in F-type mtDNA [10]. However, the putative control region or control regions of H-type mtDNAs have not been characterized. This is explored in the present study using data from Breton et al. [10] and the novel H-type mtDNA sequence presented herein. In addition, in silico analyses of H-ORFs, F-ORFs, and M-ORFs are performed to further explore the transition from gonochorism to hermaphroditism in FWMs with a focus on the topology of putative proteins associated with the orfs 
and the potential subcellular localizations and/or transport pathways of these proteins within the cell. Previous research has investigated the presence of TMs and SPs in these sex-specific ORFs; herein we extend these studies with the inclusion of additional FWM taxa and additional analyses to investigate the specific presence of SCSs and the topology of putative TMs.

\section{Methods}

\section{Sequencing the complete mitochondrial genome of} Anodonta cygnea

Anodonta cygnea samples were obtained from the German shore of Lake Konstanz and stored in AllProtect $^{\oplus}\left(\right.$ QIAGEN $\left.^{\mathrm{Tx}}\right)$. Genomic DNA was extracted using a modified saturated $\mathrm{NaCl}$ protocol [8, 16, 17]. Initial PCRs with modified Folmer primers [18] were conducted to sequence cox1 (LC022me2 5'-GGTCAA CAAAYCATAARGATATTGG-3' and HC0700dy2 5'TCAGGGTGACCAAAAAAYCA-3' [19, 20]). Primers developed by S. Palumbi were used to amplify rrnl (i.e. 16 SBr 5'-CCGGTCTGAACTCAGATCACGT-3' and 16 Sar 5'-CGCCTGTTTATCAAAAACAT-3' [21]). Longrange polymerase chain reactions (LR-PCRs) were used to amplify two large segments of the mitochondrial genome (approximately $6 \mathrm{~kb}$ and $9 \mathrm{~kb}$ ) using speciesspecific primers designed using Primer3 Version 4.0.0 [22] (Table 1).

LR-PCRs were conducted using a Phusion ${ }^{\circledR}$ HighFidelity DNA Polymerase Kit (New England BioLabs Inc.). Thermocycling conditions were as follows: activation at $98^{\circ} \mathrm{C}$ for $30 \mathrm{~s}$ followed by 35 cycles of $98^{\circ} \mathrm{C}$ for $15 \mathrm{~s}, 64^{\circ} \mathrm{C}$ for $40 \mathrm{~s}$, and $72^{\circ} \mathrm{C}$ for $7 \mathrm{~min}$, and a final extension at $72^{\circ} \mathrm{C}$ for $10 \mathrm{~min}$. LR-PCR products were sequenced using PacBio RSII sequencing technology and reads were assembled with de novo assembly [23] at the McGill University and Génome Québec Innovation Centre, Montréal, Canada. The resulting circular mtDNA genome (GenBank accession MF781083) was annotated for protein-coding and RNA genes using MITOS WebServer revision 917 [24] and validated using MFannot [25]. Putative tRNA genes were further confirmed using tRNAScan-SE 2.0 [26]. The gene order of the mitochondrial genome was visualized using Geneious version 9.1.7 [27]. Images of tRNA secondary

Table 1 Species specific primers used to amplify mtDNA of Anodonta cygnea using LR-PCR. FOR = forward, REV = reverse, $\mathrm{Tm}=$ melting temperature

\begin{tabular}{llll}
\hline Primer Name & Sequence $5^{\prime}-3^{\prime}$ & $\operatorname{Tm}\left({ }^{\circ} \mathrm{C}\right)$ & Target Gene \\
\hline ACP3 short FOR & CCAGCTAAAACAGGCAAAGC & 63.7 & $\mathrm{cox1}$ \\
ACP3 short REV & CGGGGTCTTTCGTCTACCT & 64.2 & $\mathrm{rrnl}$ \\
ACP3 long FOR & CCTCGATGTTGGCTTAAGGA & 64.0 & $\mathrm{rrnl}$ \\
ACP3 long REV & GGAGGTATTGGGGGATGAT & 63.6 & $\mathrm{cox1}$ \\
\hline
\end{tabular}

structures were generated by the MITOS WebServer revision 917 [24].

\section{Determining potential control regions of Anodonta cygnea mtDNA}

URs were assessed for characteristics of control regions as follows. Putative secondary structures were identified using the mfold webserver [28] and results were visualized using VARNA version 3.93 [29]. URs were run through REPFIND Webserver 4.09 [30] to check for tandem repeats. A-T nucleotide content was checked with Geneious [27]. A comparison of A. cygnea URs with those of the closely-related gonochoric species $A$. anatina, as well as with the URs of the gonochoric species Utterbackia peninsularis and the hermaphroditic species $U$. imbecillis was conducted in Geneious to calculate sequence similarity. Sequence similarity of the UR nad5trnQ was determined between $\mathrm{H}$-type and closely related F-type mitochondrial genomes by aligning H-type and F-type pairs in Geneious [27] using a ClustalW plugin and default parameters.

\section{Assessment of a putative translocation of a portion of nad5}

As will be detailed below, our analysis of URs in $A$. cygnea suggested an unusual translocation of a portion of the nad5 gene. To determine if the translocated segment corresponded to a generally less conserved region of the gene, we quantified the degree of sequence conservation along the protein by (1) aligning NAD5 amino acid sequences for $A$. anatina to the amino acid sequences of two distantly-related bivalves (Modiolus modiolus [GenBank accession: KX821782] and Meretrix lusoria [GenBank accession: ACV92129.1]), and (2) by aligning this same $A$. anatina sequence to two other species for which NAD5 sequence is well-annotated and structurally well-resolved (Homo sapiens [UniProtKB code: P03915] and Bos taurus [Protein Data Bank (PDB) ID: 5LDW Chain L]). Geneious [27] was used to: (1) generate the alignment of amino acid sequences using ClustalW and a BLOSUM cost matrix, (2) annotate the region corresponding to the nad5 translocation in $A$. cygnea, and (3) create subsequent sliding window graphs (window size $=50 \mathrm{bp}$ ) of the above sequences' shared identity values. Note that in Geneious, shared identity values are determined by calculating the number of pairwise combinations that match out of the total number of possible pairwise combinations in the alignment [27]. Regarding NAD5, we attempted to determine if the translocated region corresponds to a segment outside of this protein's main active site. Geneious [27] was used to map the putative translocated NAD5 segment onto the tertiary/quaternary structure of the Respiratory Complex 1 for Bos taurus. The 3D structure of Respiratory 
Complex 1 was retrieved in Geneious using its PDB ID: 5LDW (data based on the structures reported by [31]). To ensure that the amino acid chain within Respiratory Complex 1 that corresponds to NAD5 was correctly selected for analysis, sequence for NAD5 from $A$. anatina was aligned to protein sequence in the RCSB PDB using BLAST [32-34]. This protein BLAST analysis also served as a complementary test for our annotation of NAD5. NAD5's protein features were assigned in our figures using the annotations reported for $H$. sapiens NAD5 (UniProtKB code: P03915) at the website InterPro (an EMBL-EBI online tool, [35]).

The presence of a translocated portion of the nad5 gene was also tested through PCR in two additional A. cygnea individuals from the same population. PCRs were run using Platinum ${ }^{\circ}$ Blue PCR SuperMix with primers Tnad5FOR1 (5-TCCATTCATCCCAAATCTAAATGC-3) and Tnad5REV1 (5- AGAGTATGGGTTGATGTAAATGGGT-3), which flank the proposed translocated region. Thermocycling conditions were: activation at $94^{\circ} \mathrm{C}$ for $4 \mathrm{~min}$ followed by 39 cycles of $94^{\circ} \mathrm{C}$ for $1 \mathrm{~min}, 50^{\circ} \mathrm{C}$ for $1 \mathrm{~min}$ and $72^{\circ} \mathrm{C}$ for $1 \mathrm{~min}$, and a final extension at $72^{\circ} \mathrm{C}$ for $6 \mathrm{~min}$. PCR products were sequenced using Sanger sequencing technology at the McGill University and Génome Québec Innovation Centre, Montréal, Canada. Sequencing results were aligned using the Clustal Omega webserver [36].

Nad5 sequences were extracted from 12 complete mitochondrial genomes of freshwater mussels [see Additional file 1 for a list of sequence accession numbers]. All 12 nad5 were mapped to the putative translocated nad5 portion of $A$. cygnea and extracted in Geneious [27] using the Map to Reference function with the "highest sensitivity" parameter. An alignment of all 13 complete nad5, and an alignment of all extracted sequences that were mapped to the translocated portion was conducted in MEGA7 [37] to produce two separate alignment files. Each alignment file was tested for purifying selection, positive selection and strict neutrality using MEGA7 [37] with the Kumar method (available within the MEGA7 package; [37]) with 1000 bootstraps and pairwise deletion.

\section{In silico analysis of $\mathrm{H}-\mathrm{ORF}, \mathrm{F}-\mathrm{ORF}$ and $\mathrm{M}-\mathrm{ORF}$ sequences}

H-ORFs were assessed for SPs using the TargetP 1.1 [38] server with default settings. If a putative SP was detected using TargetP, then the SignalP 4.1 server [39] was used to further characterize the validity of the potential SP using the "sensitive" D-cutoff value. As an alternative strategy for maximizing the detection of SPs, H-ORFs were run through SignalP a second time with the method set to exclude the possibility of TMs. Subcellular localizations (e.g. cell membrane, cytoplasm, nucleus, etc.) of H-ORFs were predicted using iLoc-Animal [40] and Euk-mPLoc 2.0 (Cell-PLoc 2.0 package) [41]. M-
ORFs and F-ORFs were also assessed for SPs and subcellular localization. Finally, all ORFs were assessed for signal cleavage sites (SCS) using Geneious [27] and TargetP [38]. For comparative purposes, an alignment of an $A$. anatina F-ORF (GenBank Accession YP008802631) and an $A$. cygnea $\mathrm{H}-\mathrm{ORF}$ was run and visualized in Geneious using default parameters (i.e. a BLOSUM cost matrix, a gap open cost of 10 and a gap extend cost of 0.1). Hydrophobicity plots of these ORFs were also produced using a Kyte-Doolittle scale [42]. An additional file provides a list of all GenBank accession numbers for the F-, H-, and MORFs used in this study [see Additional file 2].

\section{Mapping of predicted transmembrane domain properties on phylogenetic trees of the F-types, $\mathrm{H}$-types and M-types} For the purpose of mapping properties of the predicted TMs of the F-type, H-type and M-type open reading frames in an evolutionary context, phylogenetic trees were produced based on cox 1 sequences of FWMs obtained from GenBank. An addititional file provides a list of all GenBank accession numbers for these data [see Additional file 3]. Basing these trees on $\operatorname{cox} 1$ sequence data (rather than on complete genomes or multiple genes) allowed us to maximize the number of $\mathrm{F}-, \mathrm{H}$ - and M-ORF sequences that could be included in the analysis (cox1 sequence data is readily available for many FWMs). F- and H-type cox 1 sequences were aligned using ClustalW [43] with default parameters (i.e. International Union of Biochemistry cost matrix, a gap open cost of 15 and a gap extend cost of 6.66). M-type sequences were aligned separately in the same way. A Tamura-Nei model of nucleotide substitution [44] was used as per the recommendation from the model test algorithm implemented in MEGA 7.0.16 [37]. Separate Bayesian inference (BI) F-/H-type and M-type trees, respectively, were produced using BEAUti and BEAST version 2.4.6 [45] with a Yule speciation process [46] and 100 million Markov chain Monte Carlo (MCMC) steps [47] with samples taken every 1000 steps. A burn in of $10 \%$ was performed on the resulting trees. Tree samples were visualized using DensiTree [48] included with BEAST version 2.4 .6 package [45], and compiled into one "best" topology using TreeAnnotator version 1.4 [49] and viewed using FigTree version 1.4.3 [50]. TM domain predictions for the $\mathrm{F}-, \mathrm{H}$ - and $\mathrm{M}$-ORFs were run using the transmembrane Hidden Markov Model (TMHMM) version 0.9 in a Geneious plugin and using the TMHMM Server version 2.0 [51]. These programs determine potential TM locations, number of potential TMs, and their orientation through the membrane (i.e. topology). Mesquite 3.2 [52] was used to assess the ancestral state and most parsimonious reconstruction of transitions for characters such as different TM topology and numbers of TMs present in an ORF. 


\section{Results}

General features of the mtDNA of the hermaphroditic swan mussel, Anodonta cygnea

The mtDNA of Anodonta cygnea contains the 13 proteincoding genes, and the two rRNA and 22 tRNA genes typically found in FWM mtDNAs (Fig. 1) and other animal mtDNAs [53]. This genome has the same gene order as the F-type of $A$. anatina. Similar to A. anatina [54], both trnS1 and trnS2 are missing a dihydrouridine arm [see Additional file 4], which is also the case in other FWMs (e.g. Lampsilis ornata) [55] and members of the order Mytiloida [56]. A fourteenth putative gene, a proposed $h$ orf, is located between nad2 and trnE, the same location as the $f$-orf in A. anatina. At $15,607 \mathrm{bp}$, the size of the genome is typical of FWMs, and there are no obvious large duplication events. However, the putative $h$-orf of $A$. cygnea does not possess the features associated with most h-orfs described by Breton et al. [13]. For example, the putative $A$. cygnea H-ORF, at 34 amino acids, is shorter than most H-ORFs (which have a mean of $161.8, n=5$ ). It also does not possess any repetitive DNA sequence motifs as found in several other $h$-orfs. The hydrophobicity profile of the H-ORF is not strongly similar to that of the F-ORF of $A$. anatina [57], which is a previously identified general characteristic of H-ORF/F-ORF comparisons [13]. An additional file shows the hydrophobicity plots of these ORFs [see Additional file 5].

\section{Characterization of URs in A. cygnea and other H-type mtDNAs}

All available $\mathrm{H}$-type mitochondrial genomes shared the same three principal URs found in other FWM F-type mitochondrial genomes [10], these are outlined in Table 2. No "H-specific URs" have been found in H-type mtDNAs compared to F-type mtDNAs. Based on an assessment of all shared URs in H-type mtDNAs (Table 2), the $\operatorname{trn} F-n a d 5$ region is less likely to be the main control region due to its small size; however, in all instances this region does exhibit putative secondary structures. $\mathrm{H}$-type URs rarely contain repeat units, but do tend to have high A-T content. The percent sequence divergence between the F-type and M-type mtDNAs proposed control region (i.e. the region between nad5-trnQ) [10] ranges from 43 to $50 \%$. The percent sequence divergence between nad5-trnQ of F-type mitochondrial genomes and an available closely related F-type is 48-66\% [Additional file 6]. The $\operatorname{trn} F-n a d 3$ region of $A$. cygnea possesses several potential secondary structures including one with strong similarity to a typical metazoan mitochondrial origin of replication (Fig. 2) [10].

An ORF was identified in the trnF-nad5 UR of $A$. cygnea for which a BLAST search $[32,58]$ was conducted using both the nucleotide and putative amino acid sequences. The nucleotide search had one alignment hit with $93 \%$ identity and $35 \%$ coverage of the

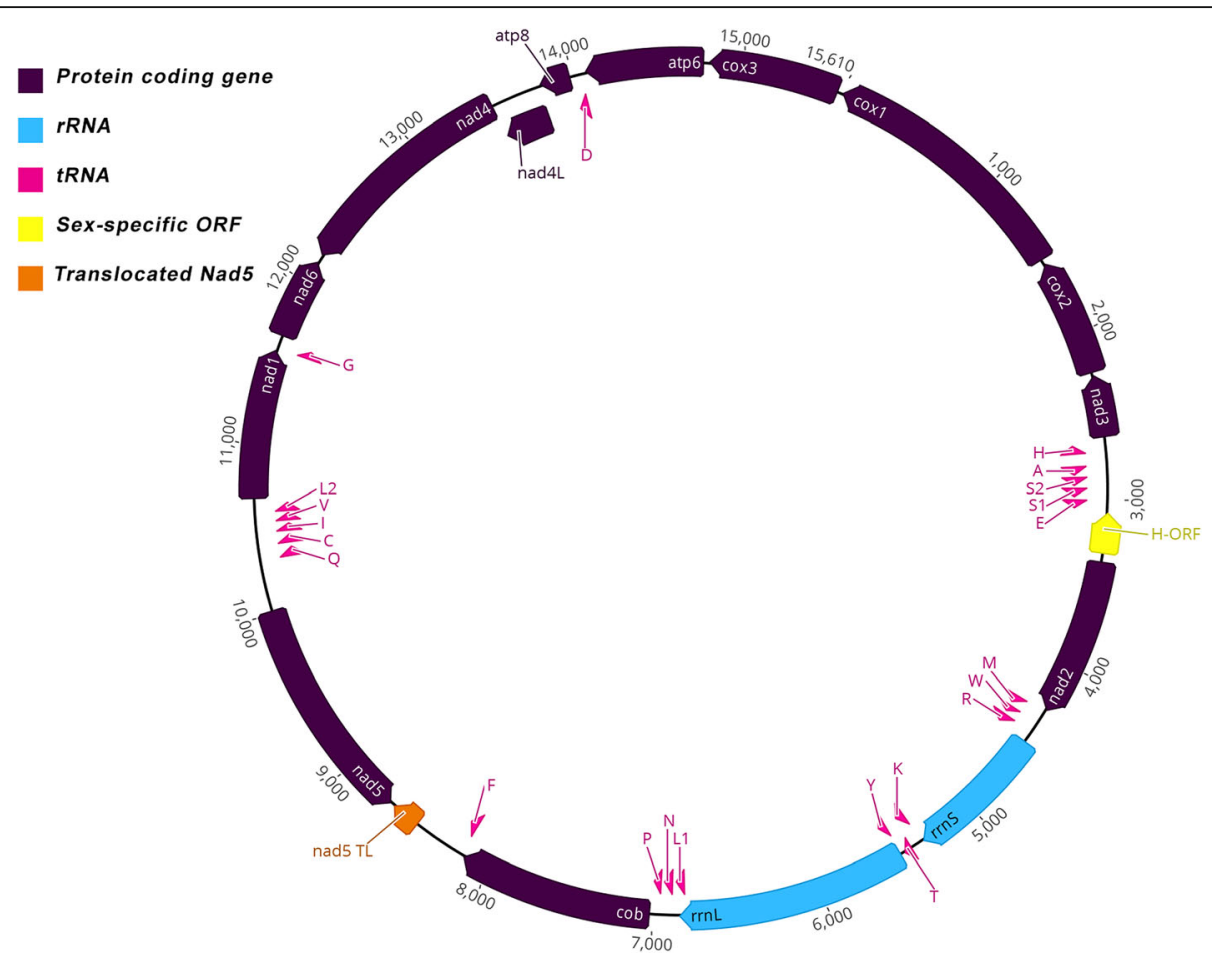

Fig. 1 Gene order and relative size of hermaphroditic freshwater mussel (order Unionoida) Anodonta cygnea H-type mitochondrial genome, with annotated tRNAs and rRNAs. Image generated in Geneious [22] 
Table 2 Assessment of hermaphroditic (H-type) Unionoid URs for presence of typical control region properties

\begin{tabular}{|c|c|c|c|c|c|}
\hline Species & UR & Length (bp) & A-T content (\%) & No. of repeats units & Potential Secondary structures \\
\hline \multirow[t]{3}{*}{ Anodonta cygnea } & $n a d 3-\operatorname{trn} H+\operatorname{trn} A$ & 153 & 80.3 & 0 & Stem loop and hairpin structure \\
\hline & nad5-trnQ & 291 & 66.5 & 0 & Stem loop \\
\hline & $\operatorname{trnF-nad5}$ & 442 & 74.9 & 0 & Stem loop and hairpin structure \\
\hline \multirow[t]{3}{*}{ Lasmigona compressa } & $n a d 3-\operatorname{trn} H+\operatorname{trn} A$ & 195 & 79.0 & 0 & Stem loop \\
\hline & nad5-trnQ & 208 & 74.0 & 1 & Stem loop and hairpin structure \\
\hline & $\operatorname{trnF-nad5}$ & 55 & 80.0 & 0 & Stem loop and hairpin structure \\
\hline \multirow[t]{3}{*}{ Margaritifera falcata } & $n a d 3-\operatorname{trnH}+\operatorname{trn} A$ & 250 & 67.2 & 0 & Stem loop and hairpin structure \\
\hline & nad5-trnQ & 171 & 77.2 & 0 & Stem loop and hairpin structure \\
\hline & trnF-nad5 & 47 & 83.0 & 0 & Stem loop \\
\hline \multirow[t]{3}{*}{ Toxolasma parvus } & $n a d 3-\operatorname{trn} H+\operatorname{trn} A$ & 300 & 65.3 & 0 & Stem loop and hairpin structure \\
\hline & nad5-trnQ & 301 & 61.1 & 0 & Stem loop and hairpin structure \\
\hline & $\operatorname{trnF-nad5}$ & 19 & 89.5 & 0 & Stem loop \\
\hline \multirow[t]{3}{*}{ Utterbackia imbecillis } & $n a d 3-\operatorname{trn} H+\operatorname{trn} A$ & 285 & 83.2 & 1 & Stem loop \\
\hline & nad5-trne & 152 & 76.3 & 1 & Stem loop \\
\hline & $\operatorname{trnF-nad5}$ & 33 & 90.9 & 0 & Stem loop \\
\hline
\end{tabular}

query sequence matching $U$. peninsularis (HM856636) within its nad5 gene $\left(\mathrm{E}\right.$-value $\left.=6 \times 10^{-16}\right)$. The protein search had 49 alignment hits, with Lasmigona compressa (another hermaphroditic FWM) being the top hit with $100 \%$ query coverage, and a $68 \%$ identity similarity (Evalue $\left.=6 \times 10^{-16}\right)$. Among the 49 hits, query coverage ranged from 90 to $100 \%$ and the percent identity ranged from 46 to $68 \%$. These results indicate that the identified ORF is likely derived from the nad5 gene. As such, the annotated nad5 from $A$. cygnea and A. anatina were compared to elucidate how the ORF is related to the nad5 gene. An alignment of NAD5 amino acid sequences from $A$. cygnea and $A$. anatina indicates low sequence similarity beyond residue number 420 [for the alignment, see Additional file 7a]. NAD5 in A. cygnea is 448 amino acids in length, whereas that of $A$. anatina is 578 amino acids long. Alignment of the putative translocated portion of NAD5 from A. cygnea shows that it is most similar to residue 452 to 513 of the complete nad5 gene from A. anatina [see Additional file 7b]. The predicted ORF protein from the $A$. cygnea trnF-nad5 UR has a percent identity of $61.3 \%$ when aligned with NAD5 of A. anatina. At $1344 \mathrm{bp}$, the putative nad5 gene of $A$. cygnea as annotated by Geneious is considerably smaller than other previously annotated FWM nad5 genes [54, 55, 59-62]. The implication of the potential translocation of a part of nad5 within the A. cygnea mtDNA is addressed below.

\section{Anodonta cygnea may have a translocated portion of nad5}

Given that it appears that an internal region of nad5 has been translocated in A. cygnea, we further assessed

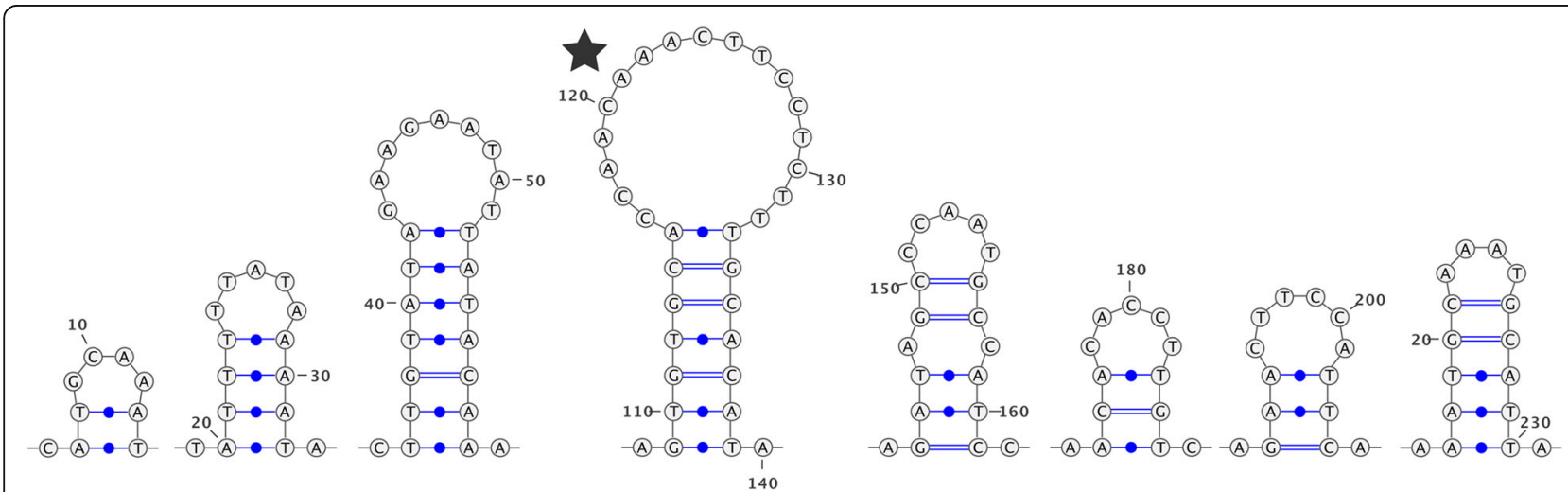

Fig. 2 Example excerpt sequence from a potential control region of Anodonta cygnea within a non-coding segment between trnF and nad5. Potential secondary structures are shown, a star indicates a potential origin of replication based on structural similarities to the classic origin of replication in metazoan control regions. Note that not all secondary structures within this region are pictured. Image generated in VARNA [24] 
whether the translocated sequence represents a portion of NAD5 that is generally less conserved, or if the translocated region contains any portion of the active site of the protein. It appears likely that the translocated portion of NAD5 is not under strong selection as the nucleotide sequence of this portion within other FWM species ranges from only an interspecific percent identity of $45.2-56.6 \%$, except in the unusual case of $L$. compressa (another hermaphrodite), which is $83 \%$ similar to this portion in A. cygnea. BLAST searches of this stretch of NAD5 with other FWM species resulted in similar percent identities among species, whereas the percent identity of NAD5 overall (including this region) ranged from $81.7-88.6 \%$ among the same set of FWM species. A multiple sequence alignment of bivalve NAD5 amino acids showing both a sliding window of identity along with the approximate location of the translocated NAD5 portion annotated, also suggests that this region is not especially well conserved within bivalves (Fig. 3a [upper plot]). Furthermore, when this analysis is expanded to include a comparison to two other metazoans outside Bivalvia for which NAD5's protein structure and functional regions have been more thoroughly classified/annotated (e.g. Homo sapiens and Bos taurus), through these comparisons we conclude that the translocated region is from a less conserved portion of the gene (Fig. 3a [lower plot]). When the translocated segment is mapped onto the structure of NAD5 (also known as "Chain L", and which was found to be A. cygnea's closest RCSB protein data bank alignment hit to an amino acid chain within

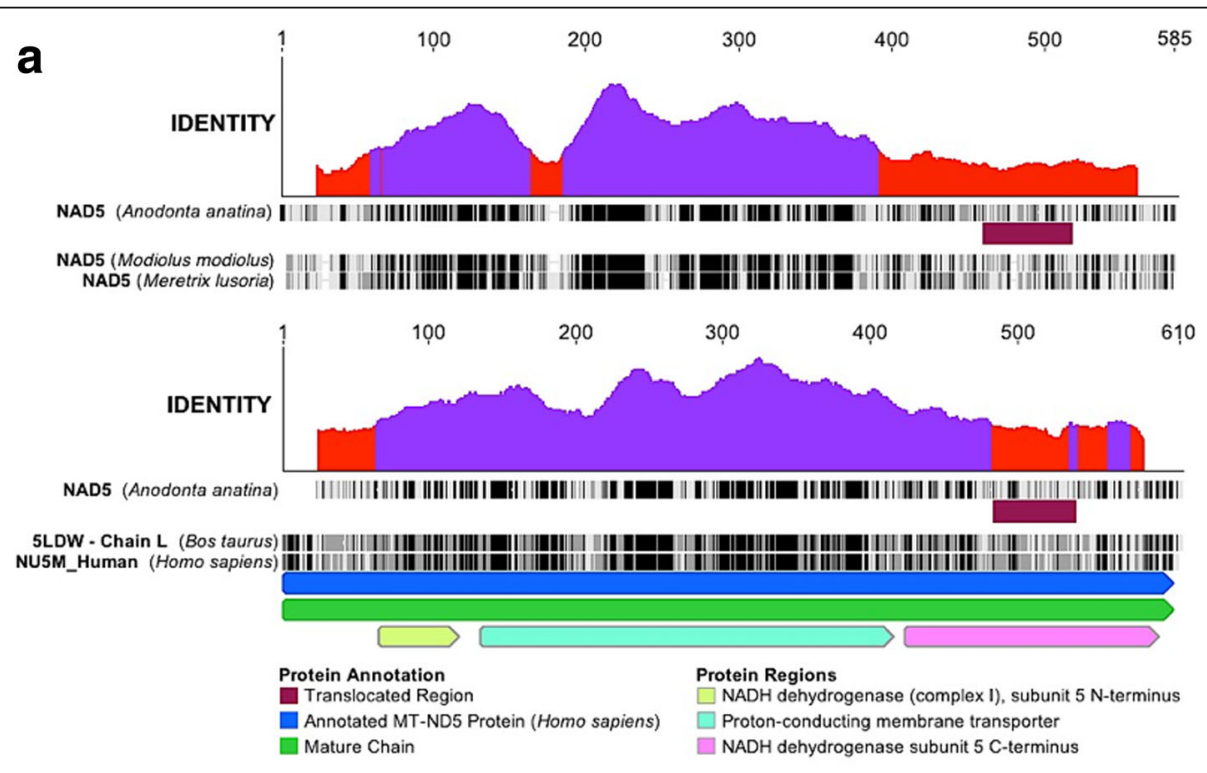

b
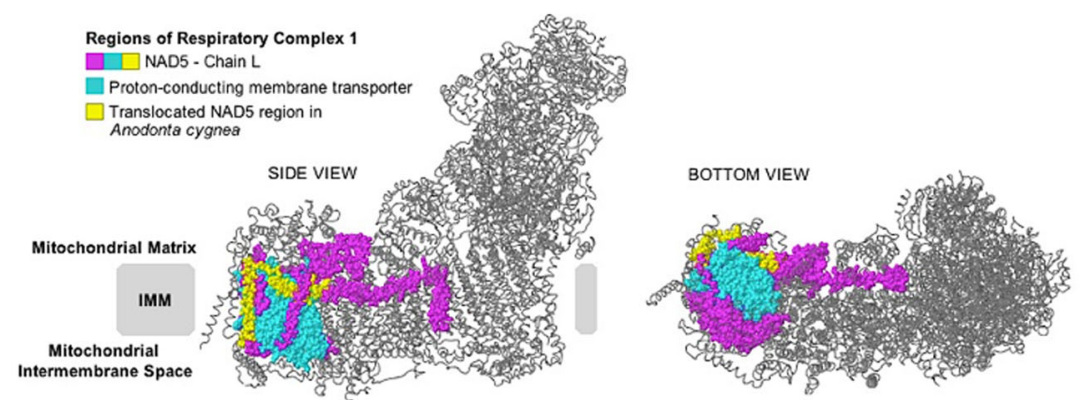

Fig. 3 Analysis of translocated nad5 region in Anodonta cygnea. a Upper plot shows NAD5 protein alignment for members of family Unionoida (Anodonta anatina; KF030964), Mytiloida (Modiolus modiolus; KX821782) and Veneroida (Meretrix lusoria; ACV92129). Lower plot shows the same A. anatina sequence aligned to Homo sapiens and Bos taurus NAD5 sequences; UniProtKB code P03915 and PDB ID: 5LDW Chain L, respectively. In both upper and lower plots the region corresponding to the partial nad5 translocation in A. cygnea is annotated. Functional regions are coloured according to the legend and descriptions provided in InterPro for H. sapiens NAD5 (accession P03915). Amino acids in alignments are colour coded by similarity (black $=100 \%$ similar, dark gray $=80-100 \%$ similar, light grey $=60-80 \%$ similar, white $=<60 \%$ similar). Identity graphs were generated with a sliding-window size of $50 \mathrm{bp}$, furthermore red and purple colours along plots represent positions that have very low identities $(30 \%)$, and less than complete identities $(30 \%$ to $<100 \%)$, respectively. See methods for an explanation of how similarity was calculated. Images were generated in Geneious [22]. b Partial nad5 translocation in A. cygnea relative to the 3-dimentional structure of Respiratory Complex 1 (PDB ID: 5LDW). In particular, NAD5 (amino acid Chain L) is coloured. Note that structures are based on work in [26] 
the $B$. taurus Respiratory Complex 1 structure [E-value = $\left.9.29 \times 10^{-68}\right]$ ) [32], it is clear that the segment corresponds to a portion of NAD5 that is outside the protein's main proton-conducting membrane transporter region (Fig. 3b) [31, 35].

Finally, we tested strict-neutrality of both the (a) complete nad5 from several FWM species [see Additional file 1 for the list of species used with accession numbers], and the (b) corresponding region of nad5 that may be translocated in A. cygnea to determine whether this region of nad5 is less conserved (Table 3). Strict neutrality was rejected $\left(\mathrm{H}_{\mathrm{o}}: \mathrm{d}_{\mathrm{n}}=\mathrm{d}_{\mathrm{s}}\right.$; two tailed test) with a $p$-value of $<0.001$ in both cases (test statistic $=-14.789$ and 6.493, for (a) and (b) respectively). Tests of purifying and positive selection (both onetailed) were conducted, based on rejecting strictneutrality, on both the complete (a) and (b) (Table 3). These results suggest that overall nad5 from FWM species is under purifying selection, whereas the region corresponding to the putative translocated portion of nad5 found in A. cygnea alone is under positive selection.

Given these results, we suggest that the area of nad5 that is translocated in A. cygnea is likely a relatively unconstrained part of the nad5 gene. This putative translocated portion of nad5 was also present in all three samples of $A$. cygnea tested (including the individual used for sequencing the complete mtDNA) [see Additional file 8], suggesting that it is a real phenomenon and not a result of a PCR or sequencing artifact.

\section{In silico analysis of H-ORFs compared to F-ORFs}

The amino acid sequence length of $\mathrm{H}$-ORFs varies considerably with the smallest reported to date being for $A$. cygnea at 34 amino acids long and the largest for $U$. imbecillis with 286 amino acids. All subsequent analyses of H-ORFs in particular are inevitably going to be affected by this variation in length. Presence of a TM were predicted for all H-ORFs were at $100 \%$, with the exception of A. cygnea (TMHMM output at $<60 \%$ ); however, we did not exclude the possibility of a TM in A. cygnea because of its relatively short length. The topology for all TM predictions included a cytoplasmic to extracellular
(C-TM-E) crossing of the membrane, although this orientation was lower for A. cygnea (63\%) and particularly low for M. falcata (22\%). TargetP detected an SP in all $\mathrm{H}$ ORFs (Reliability class $(\mathrm{RC})=1$ for A. cygnea, L. compressa, and $L$. subviridus; $\mathrm{RC}=4$ for $M$. falcata, and $T$. parvus; $\mathrm{RC}=5$ for $U$. imbecillis), and putatively assigned all H-ORF SPs as part of a secretory pathway except for $M$. falcata, which it identified as a mitochondrial SP (note: even for the lowest $\mathrm{RC}$ value of 5 , the probability of an SP being present in a sequence is greater than would be expected from a random sequence [39]). With SignalP parameters set to "sensitive" and with a potential TM presence, only $L$. subviridis came back positive for an SP. When SignalP parameters were set to exclude the possibility of a TM being present, all H-ORFs came back as positive for an SP except $M$. falcata. TMHMM webserver predicted an SP in all H-ORFs (6 total) and F-ORFs (34 total), except for the F-ORFs of $C$. monodonta and $U$. peninsularis. A SCS was predicted in 30 of $36 \mathrm{~F}$-ORFs and 4 of $6 \mathrm{H}$-ORFs.

Subcellular localization analysis predicted the following: (1) that the $T$. parvus H-ORF was localized to the cytoplasm and nucleus (iLoc-Animal) or extracellular and nucleus (EuK-mPLoc), (2) that the L. compressa H-ORF was localized to nucleus (iLoc-Animal, EuK-mPLoc), (3) that the $L$. subviridis H-ORF was localized to cellular membrane and plasma membrane (iLoc-Animal) or extracellular and nucleus (EuK-mPLoc), (4) that the M. falcata H-ORF was localized to cellular membrane and plasma membrane (iLoc-Animal) or extracellular (EuK-mPLoc), (5) and finally, that the $U$. imbecillis H-ORF was localized to cytoplasm (iLoc-Animal) or cellular membrane (EuK-mPLoc). EuKmPLoc could not assess the potential localization of the $A$. cygnea $\mathrm{H}$-ORF due to its small size, and iLoc-Animal did not localize it to any area, possibly also because of its small size. While H-ORFs overall did not have a clear subcellular localization, the F-ORF analysis using EuK-mPLoc predicted that all F-ORFs, except for $C$. monodonta and $U$. peninsularis, have an extracellular localization. Some other hits among the F-ORFs included localization to the cytoplasm, nucleus, and mitochondria; in addition, $U$. peninsularis exhibited a unique localization to endoplasmic reticulum and cell membrane. To summarize,

Table 3 Z-test of selection results for purifying selection $\left(H_{0}: d_{n}=d_{s}, H_{a}: d_{n}<d_{s}\right)$ and positive selection $\left(H_{0}: d_{n}=d_{s}, H_{a}: d_{n}>d_{s}\right)$ on alignments of (A) complete nad5 from 12 species with 543 positions, and (B) nad5 sequences corresponding to a putative translocated portion of nad5 from Anodonta cygnea (MF781083) from 12 species with 45 positions in the analysis. Alignments by ClustalW [36] and Z-tests were conducted in MEGA7 [37] using the Kumar method, 1000 bootstraps, and pairwise deletion. Statistically significant results are highlighted in bold using $a=0.05$

\begin{tabular}{|c|c|c|c|c|c|}
\hline \multicolumn{3}{|l|}{ A } & \multicolumn{3}{|l|}{ B } \\
\hline Test & P-value & Test statistic & Test & P-value & Test statistic \\
\hline Purifying selection & $<0.001$ & 14.932 & Purifying selection & 1.000 & -6.456 \\
\hline Positive selection & 1.000 & -14.495 & Positive selection & $<0.001$ & 6.842 \\
\hline
\end{tabular}


the localization of the ORF proteins, and particularly the H-ORFs, as determined by in silico analyses is highly variable. It must be noted, however, that with the exception of the H-ORFs present in the two species of Lasmigona, each of these proteins represent an independent transition from an F-ORF in a gonochoric species, to an H-ORF in a hermaphroditic species. The possible implications of the independent nature of these multiple transitions to the hermaphroditic state will be discussed below.

\section{In silico analysis of M-ORFs}

All $11 \mathrm{M}$-ORFs contained at least one potential TM with variable topologies. All M-ORFs possessed an SCS predicted by either or both Geneious and TargetP. All predicted SCS were within the potential TM hydrophobic stretch of amino acids. No clear pattern of subcellular localization hits of M-ORFs resulted from the EuKmPLoc or iLoc-Animal analyses.

\section{Determination of evolutionary pattern(s) of TM predictions and their associated topologies among the sex-associated mitochondrial ORF proteins}

The following results are based on constructing BI trees of F-/H-type or M-type cox 1 sequences and examining the trees for evolutionary patterns of $\mathrm{F}-, \mathrm{H}-$ and $\mathrm{M}$-ORF TM predictions and topologies as identified in the previous section. Almost all gonochoric and hermaphroditic species have one F-/H-ORF TM prediction with a CTM-E topology (Fig. 4), although some predictions are not at $100 \%$ likelihood for either orientation or presence of a TM $(<80 \%$ likelihood). The only major exception was the F-ORF of Ellipsaria lineolata, which potentially possesses two TMs, but the topology of these TMs was not strongly supported (correct orientation of the first TM at only $8 \%$ probability). There is no clear link between phylogenetic placement on the $\operatorname{cox} 1$ tree and the absence of an SCS predicted within the F-ORFs, except for the genus Toxolasma. A parsimonious reconstruction of both number of TMs and their topologies suggests that one TM and an extracellular-TM-cellular (E-TM-C) topology is the ancestral state of all M-type FWMs included (Fig. 5).

\section{Discussion}

The complete mtDNA genome of Anodonta cygnea is similar to other F-/H-type FWM mtDNA genomes with respect to location of protein coding genes, rRNAs, tRNAs and the H-ORF. An interesting exception is a translocation of a portion of nad5. The principal URs of A. cygnea and other $\mathrm{H}$-type mtDNAs share some, but not all, common features of F-type URs. As expected based on prior analyses of FWM H-ORFs with F-ORFs of closely related species [13], the putative $\mathrm{H}$-ORF of $A$. cygnea shows little similarity to the F-ORF of a close relative, A. anatina (Additional file 5: Figure S2). However, somewhat unexpectedly, it does not contain the common features of other H-ORFs as outlined by Breton et al. [13]. Consistent with other hermaphroditic FWMs, there is no evidence of an M-type mitochondrial genome in this population of hermaphroditic A. cygnea [6]. Further insights into the putative $\mathrm{H}-\mathrm{ORF}$ of $A$. cygnea, and molecular characterization of F-/H-ORFs of FWMs in general are discussed below.

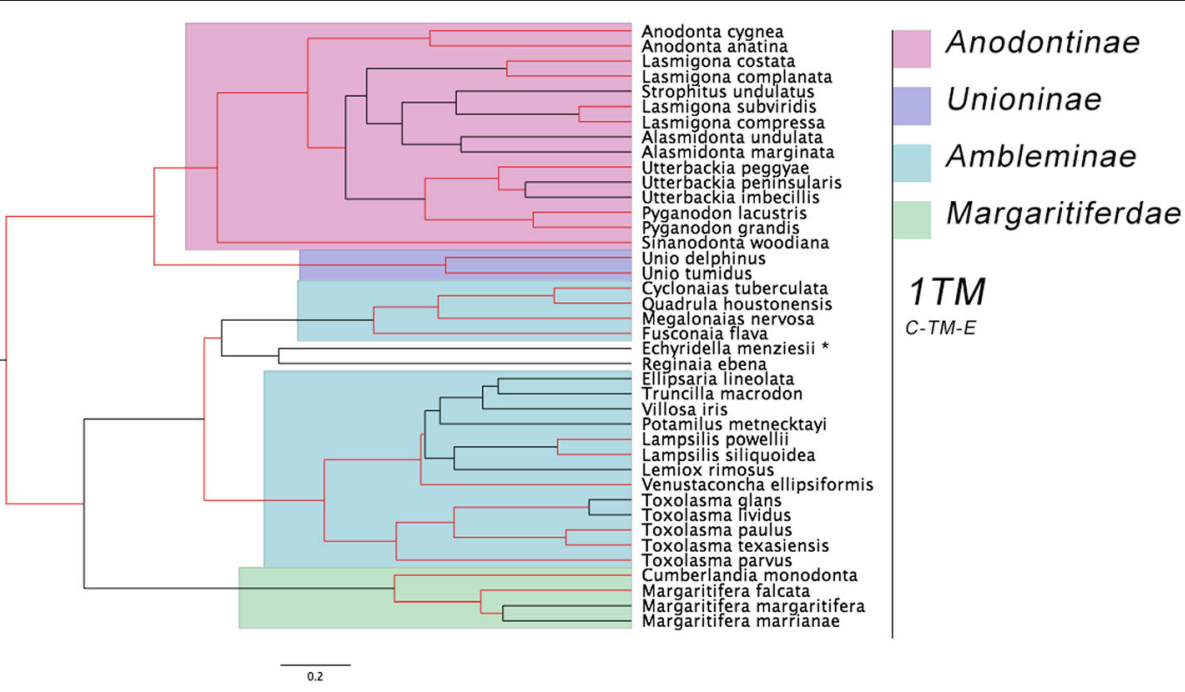

Fig. 4 F-type and H-type BI phylogenetic tree based on cox 1 DNA sequences. Posterior probabilities $>0.75$ are marked in red. A (*) denotes a species that has potential of possessing two TMs. Number of predicted transmembrane domains (TM) and their topology are shown ( $E=$ extracellular, $\mathrm{C}=$ cytoplasmic). Subfamilies are colour-coded. A single species represents subfamily Hyriidae (Echyridella menziesii), and Ambleminae (Reginaia ebena). Tree annotated in FigTree [45] 


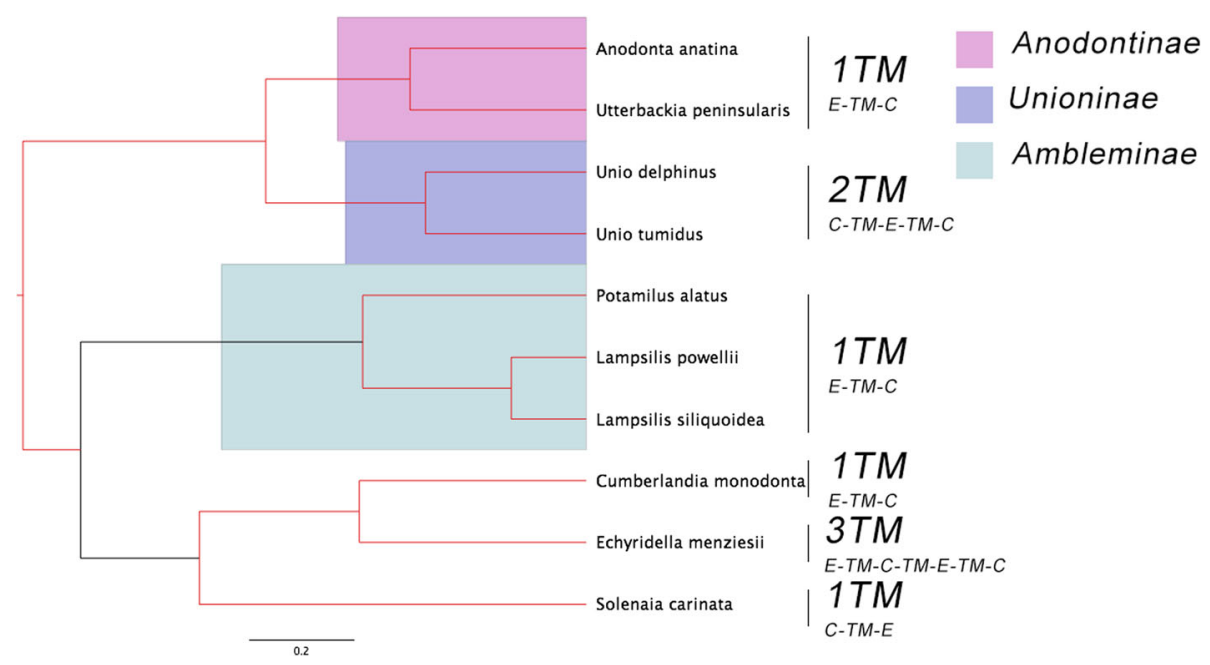

Fig. $5 \mathrm{M}$-type Bl phylogenetic tree of based on cox1 DNA sequences. Posterior probabilities $>0.75$ are marked in red. Number of predicted transmembrane domains (TM) and their topology are shown ( $E=$ extracellular, $C=$ cytoplasmic). Subfamilies are colour-coded. A single species represents subfamily Margaritiferdae (Cumberlandia monodonta), Hyriidae (Echyridella menziesii), and Gonideinae (Solenaia carinata). Tree annotated in FigTree [45]

\section{A portion of nad5 is translocated to the trnF-nad5 UR in Anodonta cygnea}

Our analysis of the trnF-nad5 UR suggested the presence of a partial nad5 translocation. Serb and Lydeard [55] previously used the same approach based on amino acid similarity to identify a translocated portion of atp8 in the F-type genome of Unio japanensis. Although nad5 does not appear to be highly modified among A. anatina, $U$. peninsularis, and $U$. imbecillis, it is possible that the region of nad5 that is translocated is less conserved. If a portion of nad5 can translocate (or the parts of nad5 flanking it) then it is possible that selective pressures are relatively unequal across the gene and that oxidative phosphorylation functions are not interrupted with the movement of this piece. This piece of nad5 could be transcribed and translated separately from the remainder of the gene given that both pieces have their own start codons. It is likely that the amino acids that form the active sites of the protein's tertiary structure would be under stronger selective constraints than neighbouring amino acids that play a primarily structural role (e.g. [63]). Regarding this point, our additional analyses mapping the translocated segment to the tertiary/quaternary structure of Respiratory Complex 1 and conducting Z-tests of selection, further support the notion that the translocation is outside the main proton-conducting region of NAD5 and that it corresponds to a region within the protein's C-terminus. Overall, we hypothesize that NAD5 of A. cygnea functions adequately with this component being translocated. $\mathrm{Fu}-$ ture research would require functional studies to test this hypothesis.

\section{Putative control region(s) appear similar among F- and} H-type mtDNAs

Although secondary structures were located within all principal URs, and within the longest unassigned region of all complete H-type mtDNAs available, it is difficult to pinpoint a main control region similar to other metazoan mitochondrial genomes. The proposition that nad5-trnQ UR [10] is the main control region of F-type mtDNA is also possible for the $\mathrm{H}$-type mtDNA; however, nad3-trnH $+\operatorname{trn} A$ also appears to be a potential candidate for the primary control region. Curiously, only three URs within five H-type complete mtDNAs (15 URs total) assessed contain repeats, which is notably different than the pattern described in Breton et al. [10]. In that study, all main URs of the seven F-type mtDNAs assessed (35 URs total) contained at least one repeat and at most 33. It is unclear why a shift from a gonochoric sexual strategy to a hermaphroditic sexual strategy would accompany a loss in repetitive sequence within URs, however, there may be something specific about repeat arrays in URs that are no longer required following this shift in reproductive strategy and the concomitant changes in processes of germ line, gonad tissue and gamete development.

Within F-type mtDNA, nad5-trnQ ranges from 202 to $450 \mathrm{bp}$ (excluding the exceptional case of $U$. japanensis at $1196 \mathrm{bp}$ ), whereas this region ranges from 152 to $291 \mathrm{bp}$ in H-type mitochondrial genomes. There does not appear to be a reduction in complete mtDNA length overall with the transition from gonochorism to hermaphroditism. Figure 2 provides an example of secondary structures, and thereby potential nucleotide motifs such as an origin of replication or transcription, occurring 
within the A. cygnea trnF-nad3 UR. However, similar structures occur within main URs from all other $\mathrm{H}$-type complete mtDNAs analyzed and this feature is therefore not unique to A. cygnea. As noted in Breton et al. [10], these URs are associated with a change in the direction of transcription within the mitochondrial genome, and an origin of transcription occurring within each would seem to make sense.

The transition from a gonochoric to hermaphroditic sexual strategy in FWMs must involve modifications to cellular processes to facilitate production of both types of gametes in one individual. If mitochondrial genes are involved in the developmental pathways that determine sex, then the F-, M, and H-type genomes may contain distinct genetic signatures that play a role in these processes. Examining the mitochondrial URs further will provide us with a better understanding of the basis of these developmental systems. However, based on the present analysis it appears that the only major difference in URs between F- and H-type mtDNAs is the loss of tandem repeats.

\section{In silico analyses of $\mathrm{H}-$, $\mathrm{F}-$, and $\mathrm{M}-\mathrm{ORFs}$}

The in silico analyses presented here agree with the findings of Mitchell et al. [11] in that (1) TMs were predicted in almost all ORFs (although their likelihood varied) and (2) SPs were predicted in almost all ORFs, but again these predictions remains somewhat less certain as "sensitive" parameters were used for this analysis. SPs possess a tripartite structure that includes a hydrophilic region, a hydrophobic region and an SCS [64]. The hydrophilic region usually consists of positively charged amino acids, varies in length [65], and generally has the highest variability of all three regions [66]. In contrast, the hydrophobic region is considered the most essential feature of an SP [67]. Although some SP predictions were uncertain, the confirmation of both a hydrophobic region and an SCS leave only the variable hydrophilic region to be confirmed; theoretically this variability (especially in these novel ORFs with a high substitution rate) could result in under-identification of the region in question. Although SPs share this tripartite structure, they do not possess a single characteristic sequence motif and instead can tolerate mutations [67, 68]. SPs target proteins to a membrane and can reach their destination through different pathways. The topology of a protein during translocation through a protein conducting channel is C-TM-E, which, interestingly, is the orientation consistently predicted for F- and H-ORFs (Fig. 4). MORFs adopt this orientation less often (Fig. 5). If the SCSs of SPs leave a significant hydrophobic region after cleavage then this structure could function as an anchor for the SP at its target destination $[65,69,70]$. The SCS locations across these novel ORFs vary in relation to their placement along the hydrophobic stretch of amino acids, therefore the number of hydrophobic amino acids remaining after cleavage is variable. Consequently, it is inconclusive if these proteins will be anchored or not at their final destination. These speculations must be investigated with future, targeted approaches. Interestingly, all known examples of TMs formed from the hydrophobic region of SPs have a viral origin [64]. Both Milani et al. [71] and Mitchell et al. [11] suggest that these ORFs could have a viral origin, although Mitchell et al. [11] and Guerra et al. [12] also suggest that the process of gene duplication and subsequent modification of a mitochondrial gene may have been responsible. Determining whether or not these orfs produce anchored proteins is relevant for testing these hypotheses regarding the origin of the orf genes.

For several FWMs, Mitchell et al. [11] identified motifs and domains of F-ORFs and M-ORFs related to cell membrane and surface anchoring, but independent in situ verification of the localization of these polypeptides would be useful. In the case of V. ellipsiformis, the FORF was associated with both cytoplasm (mitochondria and nucleoplasm) and membrane (plasma membrane), but this does not necessarily imply that these structures are the final destinations for these proteins. Multiple cleavage sites were predicted for many F-ORFs, consequently it may be possible to have both anchored and non-anchored versions of one protein. However, it should be noted that for the F-ORF of $V$. ellipsiformis only one SCS was predicted using TargetP. This SCS was located near the end of the hydrophobic stretch leaving relatively few hydrophobic amino acids to act as an anchor. It has also been suggested that SP fragments, which have been cleaved at the SCS, may have posttargeting functions and that the cleaved proteins themselves might undergo additional processing [66]. This further complicates identifying the potential function(s) of these novel ORFs given their multiple putative SPs and SCSs. To add another layer of complexity, SPs are not always cleaved, particularly in the case of some viral proteins [65]. Collectively, these factors shed some light on the variety of subcellular localization hits resulting from our in silico analyses. Furthermore, the putative localizations do not always agree with the results of in situ analyses. For example, the V. ellipsiformis F-ORF was localized as extracellular based on the in silico analysis and not predicted to occur in any of the locations identified through immunostaining [13]. The difficulty in accurately predicting functions and sub-cellular localizations, specifically, for these ORFs may be because (1) they are not homologous to any other known and previously characterized proteins, (2) they may be participating in unique functions associated with a unique phenomenon (i.e. DUI), and (3) they are produced in the 
mitochondria and migrating to the nucleus (also a unique process) and standard in silico analysis programs are not optimized to characterize such proteins. For example, the programs iLoc-Animal and EuK-mPLoc rely on the Swiss-Prot Database [40, 41, 72, 73], which is not optimized to predict localization for mitochondrial proteins that are exported to other cellular structure(s). In silico analyses could still be useful for constructing hypotheses that can tested using in situ analytical techniques such as the immunostaining conducted by Breton et al. [13]. These or similar analyses such as the electrophoretic mobility shift analyses (EMSA) conducted by Kyriakou et al. [74] to characterize interactions between proteins and mitochondrial DNA sequence motifs will be necessary to create a more complete picture of what these fascinating proteins are doing, and if they could be a component in a sex determination mechanism in bivalves possessing DUI.

\section{Putative TM topology of F-/H-ORF and M-ORFs exemplifies the variable selective constraints placed on $\mathrm{F}-, \mathrm{H}$ - and $\mathrm{M}$ - type mtDNA}

The F-type and H-type cox1 tree (Fig. 4) closely reflects the groupings suggested in Lopes-Lima et al. [75], with the exception of the position of Echyridella menziesii. However, there is not strong support for the placement of this particular species on the tree (i.e. $<75 \%$ posterior probability). It is possible that including additional genes would resolve this tree topology to more closely reflect the BI tree presented in Breton et al. [13], which was based on both cox 1 and nad1 sequences. The E. lineolata F-ORF was scored as potentially having two TMs, however, the first TM likelihood was $<80 \%$, whereas the second TM was above this threshold based on TMHMM webserver results. The topology of the putative TM was also somewhat uncertain (8\% probability of the correct topology being predicted), further reducing the likelihood of this region forming a TM. Consequently, it is likely that all the FWM species included in the F-/Htype cox 1 tree possess an ORF with a single TM domain and a C-TM-E orientation. These trees (Figs. 4 and 5) suggest that a stronger selective pressure is placed on the F-ORFs and potentially the H-ORFs to maintain a hydrophobic region that forms a TM. An analysis of a total of 40 FWM species (either female or hermaphroditic) provides a clear indication that this $\mathrm{TM}$ and its topology is being maintained across several subfamilies. In contrast, analysis of a total of 10 males suggest that the number of TMs and their topology is not maintained across several subfamilies, but may be maintained within some subfamilies. Interestingly, among closely related subfamilies on the M-type tree for the families Anodontinae and Unioninae (this study; [76]), a drastic change from one TM and an E-TM-C topology to two TMs and a C-TM-E-TM-C topology occurs. The ancestral topology of F-/H-ORFs is opposite to the ancestral topology predicted for M-ORFs. M-orf and f-orf are likely not homologous genes and are derived from separate evolutionary events $[13,71]$, it is therefore not surprising that they have different ancestral topologies. However, it is unclear if DUI or these novel ORFs appeared first as it has yet to be determined precisely what function the ORFs have, if they all have similar functions, or if some are functioning while others (i.e. H-ORFs) have lost functionality.

A comparison between these two trees exemplifies the selective constraints placed on the F-ORFs compared to the M-ORFs and similarly the selective constraints placed on F-type and M-type mtDNA of species with DUI. Although it has been shown that both FWM ORFs have a high amino acids substitution rate [10], it has also been shown that M-type mtDNA typically evolves much more quickly than F-type mtDNA in bivalves exhibiting DUI [76-78]. It is possible that selective constraints placed on F-ORFs are much higher than those placed on M-ORFs, which is illustrated by the multiple character states in this relatively small sample of FWM M-ORFs. Although patterns have emerged from the comparison of $\mathrm{H}$-ORFs and closely related F-ORFs [13], H-ORF comparisons show a high level of sequence divergence and their hydrophobicity profiles vary greatly [13]. This is particularly interesting given that the hermaphroditic reproductive mode has evolved independently multiple times within order Unionoida.

\section{Conclusions}

Overall, the transition from gonochorism to hermaphroditism appears to conserve various features of the F-type mtDNA genome during modification to the $\mathrm{H}$-type mtDNA genome. This is highlighted by our assessment of URs and our in silico analysis of F-ORFs compared to H-ORFs. To summarize, we have (1) added a fifth complete mitochondrial genome to the existing pool of data, (2) demonstrated the first potential translocated nad5 gene portion within a hermaphroditic FWM, (3) provided further evidence of signal peptides and evidence of SCS in the F-, and H-ORFs, which may be carrying out functions related to sex determination after transportation to the nucleus and (4) linked the number of TMs and their topology to FWM F- and M-type lineages. However, the putative TMs and potential SPs within M-ORFs remain unclear and inconsistent. Further exploration of potential SPs and the subcellular localization of these novel proteins (F-, M- and H-ORF) should include in situ analysis to overcome barriers associated with the novelty of these proteins within in silico analysis (e.g. using programs based on protein banks with no clear homology to F-, M- and H-ORFs). 


\section{Additional files}

Additional file 1: Table S1. Genbank accessible nad5 sequences used in this study. (PDF $68 \mathrm{~kb}$ )

Additional file 2: Table S2. Genbank accessible F-ORFs, H-ORFs, and M-ORFs used in this study. (PDF $72 \mathrm{~kb}$ )

Additional file 3: Table S3. Genbank accessible cox1 sequences used in this study [79-85] (PDF $81 \mathrm{~kb}$ )

Additional file 4: Figure S1. Predicted tRNA structures for all 22 tRNA of H-type mitochondrial DNA in A. cygnea. Left-to-right and top-tobottom order follows their placement within the annotated complete mitochondrial genome beginning at base 2668. (PDF $164 \mathrm{~kb}$ )

Additional file 5: Figure S2. Comparative analysis of an F- and H-ORF of closely related species. (A) Kyte-Doolittle hydrophobicity plots of the Anodonta anatina F-ORF (GenBank Accession YP008802631) and Anodonta cygnea $\mathrm{H}$-ORF (this study). (B) Amino acid alignment of the same A. anatina F-ORF and A. cygnea H-ORF, where black represents the same amino acid, grey is similar and white is different. (PDF $116 \mathrm{~kb}$ )

Additional file 6: Table S4. Sequence divergence of nad5-trnQ between $\mathrm{H}$-type and closely related F-type mitochondrial DNA. (PDF $43 \mathrm{~kb}$ )

Additional file 7: Figure S3. NAD5 amino acid alignment of Anodonta cygnea and Anodonta anatina. (PDF $84 \mathrm{~kb}$ )

Additional file 8: Figure S4. Nucleotide alignment of three translocated portions of nad5 from three Anodonta cygnea individuals. A.cygnea 3 is the subject of this paper (Genbank accession MF781083). (PDF $70 \mathrm{~kb}$ )

\section{Abbreviations}

BI: Bayesian inference; C-TM-E: Cytoplasmic to extracellular; DUI: Doubly uniparental inheritance; EMSA: Electrophoretic mobility shift analyses; E-TMC: Extracellular-TM-cellular; FWM: Freshwater mussels; LR: Long-range; mtDNA: mitochondrial DNA; ORFs: open reading frames; PCR: polymerase chain reaction; PDB: protein data bank; RC: reliability class; SCSs: signal cleavage sites; SPs: signal peptides; TMs: transmembrane domains; URs: unassigned regions

\section{Acknowledgments}

We thank Dr. Timothy Rawlings and Dr. Melanie Coombs for comments on an earlier version of this manuscript. We also thank Dr. Gerhard Bauer for providing samples of Anodonta cygnea.

\section{Funding}

This research was funded by NSERC Discovery grants to DTS and SB. Support for DTS to visit the lab of GB was supported by a Deutscher Akademischer Austausch Dienst Research Visit Grant (Germany) and by a McCain Foundation Visiting Professorship Grant. EEC and BMR were supported by Acadia Graduate Awards and SV was supported by an NSERC Undergraduate Student Research Award.

\section{Availability of data and materials}

The datasets generated and/or analysed during the current study are available on the GenBank repository, https://www.ncbi.nlm.nih.gov/genbank/. GenBank accession numbers are listed in Additional file 2 and Additional file 3. The GenBank accession number for the complete Anodonta cygnea mitochondrial genome referred to in the text is MF781083.

\section{Authors' contributions}

Conceived and designed research: DTS, EEC, SB and BMR. Contributed samples and council on the study species: GB. Conducted laboratory work: EEC and SV. Conducted analyses: EEC and BMR. Writing the manuscript: EEC, DTS, BMR, SB. All co-authors approved the final manuscript.

\section{Author's information}

Not applicable.

\section{Ethics approval and consent to participate}

All use of animals in this research was conducted in accordance with the guidelines of the Canadian Council on Animal Care. Field permissions were not required as the three Anodonta cygnea individuals analysed were from the collection of Dr. Gerhard Bauer, at the Institut für Biologie, Universität Freiburg, Freiburg, Germany.

\section{Consent for publication}

Not applicable.

\section{Competing interests}

The authors declare that they have no competing interests.

\section{Publisher's Note}

Springer Nature remains neutral with regard to jurisdictional claims in published maps and institutional affiliations.

\section{Author details}

${ }^{1}$ Department of Biology, Acadia University, Wolfville, NS, Canada.

${ }^{2}$ Department of Biology, Dalhousie University, Halifax, NS, Canada.

${ }^{3}$ Département de Sciences Biologiques, Université de Montréal, Montréal, QC, Canada.

Received: 23 October 2017 Accepted: 7 March 2018

Published online: 27 March 2018

\section{References}

1. Bogan AE. Freshwater bivalve extinctions (Mollusca: Unionoida): a search for causes. Integr Comp Biol. 1993;33:599-609.

2. Birky CW. Uniparental inheritance of mitochondrial and chloroplast genes: mechanisms and evolution. PNAS. 1995;92:11331-8.

3. Breton S, Beaupré HD, Stewart DT, Hoeh WR, Blier PU. The unusual system of doubly uniparental inheritance of mtDNA: isn't one enough? Trends Genet. 2007;23:465-74

4. Zouros E. Biparental inheritance through uniparental transmission: the doubly uniparental inheritance (DUI) of mitochondrial DNA. Evol Biol. 2013; 40:1-31.

5. Gusman A, Lecomte S, Stewart DT, Passamonti M, Breton S. Pursuing the quest for better understanding the taxonomic distribution of the system of doubly uniparental inheritance of mtDNA. PeerJ. 2016; https://doi.org/10. 7717/peerj.2760

6. Stewart DT, Hoeh WR, Bauer G, Breton S. Mitochondrial genes, sex determination and hermaphroditism in freshwater mussels (Bivalvia: Unionoida). In: Pontarotti P, editor. Evolutionary biology: exobiology and evolutionary mechanisms. Springer, berlin, Heidelberg; 2013. p. 245-55.

7. Garrido-Ramos MA, Stewart DT, Sutherland BW, Zouros E. The distribution of male-transmitted and female-transmitted mitochondrial DNA types in somatic tissues of blue mussels: implications for the operation of doubly uniparental inheritance of mitochondrial DNA. Genome. 1998;41:818-24.

8. Dalziel AC, Stewart DT. Tissue-specific expression of male-transmitted mitochondrial DNA and its implications for rates of molecular evolution in Mytilus mussels (Bivalvia: Mytilidae). Genome. 2002;45:348-55.

9. Venetis C, Theologidis I, Zouros E, Rodakis GC. No evidence for presence of maternal mitochondrial DNA in the sperm of Mytilus galloprovincialis males. Proc Biol Sci. 2006;273:2483-9.

10. Breton S, Beaupré HD, Stewart DT, Piontkivska H, Karmakar M, Bogan AE, Blier PU, Hoeh WR. Comparative mitochondrial genomics of freshwater mussels (Bivalvia: Unionoida) with doubly uniparental inheritance of mtDNA: gender-specific open reading frames and putative origins of replication. Genetics. 2009:183:1575-89.

11. Mitchell A, Guerra D, Stewart D, Breton S. In silico analyses of mitochondrial ORFans in freshwater mussels (Bivalvia: Unionoida) provide a framework for future studies of their origin and function. BMC Genomics. 2016;17:597.

12. Guerra D, Plazzi F, Stewart DT, Bogan AE, Hoeh WR, Breton S. Evolution of sex-dependent mtDNA transmission in freshwater mussels (Bivalvia: Unionida). Sci Rep. 2017;7:1551

13. Breton S, Stewart DT, Shepardson S, Trdan RJ, Bogan AE, Chapman EG, Ruminas AJ, Piontkivska H, Hoeh WR. Novel protein genes in animal mtDNA: a new sex determination system in freshwater mussels (Bivalvia: Unionoida)? Mol Biol Evol. 2011;28:1645-59. 
14. Breton S, Milani L, Ghiselli F, Guerra D, Stewart DT, Passamonti M. A resourceful genome: updating the functional repertoire and evolutionary role of animal mitochondrial DNAs. Trends Genet. 2014;30:555-64.

15. Passamonti M, Ghiselli F. Doubly uniparental inheritance: two mitochondrial genomes, one precious model for organelle DNA inheritance and evolution. DNA Cell Biol. 2009;28:79-89.

16. Miller SA, Dykes DD, Polesky H. A simple salting out procedure for extracting DNA from human nucleated cells. Nucleic Acids Res. 1988;16: 1215

17. Seutin G, White BN, Boag PT. Preservation of avian blood and tissue samples for DNA analyses. Can J Zool. 1991;69:82-90.

18. Folmer O, Black M, Hoeh WR, Lutz R, Vrijenhoek RDNA. Primers for the amplification of mitochondrial cytochrome c oxidase subunit I from diverse metazoan invertebrates. Mol Mar Biol Biotechnol. 1994;3:294-9.

19. Walker JM, Bogan AE, Bonfiglio EA, Campbell DC, Christian AD, Curole JP, Harris JL, Wojtecki RJ, Hoeh WR. Primers for amplifying the hypervariable, male-transmitted COII-COI junction region in amblemine freshwater mussels (Bivalivia: unionoidea: ambleminae). Mol Ecol Notes. 2007;7:489-91.

20. Walker JM, Curole JP, Wade DE, Chapman EG, Bogan AE, Watters GT, Hoeh WR. Taxonomic distribution and phylogenetic utility of gender-associated mitochondrial genomes in the Unionoida (Bivalvia). Malacologia. 2006;48: 265-84

21. Palumbi S, Martin A, Romano WO, McMillan L, Stice L, Grabowski G. The simple fools guide to PCR, version II. Honolulu: University of Hawaii; 1991.

22. Untergasser A, Cutcutache I, Koressaar T, Ye J, Faircloth BC, Remm M, et al. Primer3-new capabilities and interfaces. Nucleic Acids Res. 2012;40:e115.

23. Rhoads A, Au KF. PacBio sequencing and its applications. Genomics Proteomics Bioinformatics. 2015:13:278-89

24. Bernt M, Donath A, Jühling F, Externbrink F, Florentz C, Fritzsch G, Pütz J, Middendorf M, Stadler PF. MITOS: Improved de novo metazoan mitochondrial genome annotation. Mol Phylogenet Evol. 2013;69:313-9.

25. Beck N, Lang BF. MFannot, organelle genome annotation webserver. Canada: Université de Montréal QC; 2010. http://megasun.bch.umontreal.ca/ RNAweasel/. Accessed 31 May 2017

26. Lowe TM, Eddy SR. tRNAscan-SE: a program for improved detection of transfer RNA genes in genomic sequence. Nucleic Acids Res. 1997;25: 955-64.

27. Kearse M, Moir R, Wilson A, Stones-Havas S, Cheung M, Sturrock S, et al. Geneious basic: an integrated and extendable desktop software platform for the organization and analysis of sequence data. Bioinformatics. 2012;28: $1647-9$

28. Zuker M. Mfold web server for nucleic acid folding and hybridization prediction. Nucleic Acids Res. 2003;31:3406-15.

29. Darty K, Denise A, Ponty Y. VARNA: interactive drawing and editing of the RNA secondary structure. Bioinformatics. 2009;25:1974-5.

30. Benson $\mathrm{G}$. Tandem repeats finder: a program to analyze DNA sequences, Nucleic Acids Res. 1999:27:573-80.

31. Zhu J, Vinothkumar KR, Hirst J. Structure of mammalian respiratory complex I. structure of mammalian respiratory complex I. Nature. 2016;536:354-8.

32. Altschul SF, Gish W, Miller W, Myers EW, Lipman DJ. Basic local alignment search tool. J Mol Biol. 1990;215:403-10.

33. Berman HM, Westbrook J, Feng Z, Gilliland G, Bhat TN, Weissig H, et al. The protein data bank. Nucleic Acids Res. 2000;28:235-42.

34. Rose PW, Prlić A, Altunkaya A, Bi C, Bradley AR, Christie CH, et al. The RCSB protein data bank: integrative view of protein, gene and 3D structural information. Nucleic Acids Res. 2017:45:D271-81.

35. Finn RD, Attwood TK, Babbitt PC, Bateman A, Bork P, Bridge AJ, et al. InterPro in 2017-beyond protein family and domain annotations. Nucleic Acids Res. 2017:45:D190-9.

36. Sievers F, Wilm A, Dineen D, Gibson TJ, Karplus K, Li W, et al. Fast, scalable generation of high-quality protein multiple sequence alignments using Clustal omega. Mol sys. Biol. 2011;7:539.

37. Kumar S, Stecher G, Tamura K. MEGA7: molecular evolutionary genetics analysis version 7.0 for bigger datasets. Mol Biol Evol. 2016;33:1870-4.

38. Guda C, Subramaniam S. TARGET: a new method for predicting protein subcellular localization in eukaryotes. Bioinformatics. 2005;21:3963-9.

39. Emanuelsson O, Brunak S, von Heijne G, Nielsen H. Locating proteins in the cell using TargetP, SignalP and related tools. Nat Protoc. 2007;2:953-71.

40. Lin WZ, Fang JA, Xiao X, Chou KC. iLoc-animal: a multi-label learning classifier for predicting subcellular localization of animal proteins. Mol BioSyst. 2013;9:634.
41. Chou KC, Shen HB. Cell-PLoc 2.0: an improved package of web-servers for predicting subcellular localization of proteins in various organisms. Nat Sci. 2010;2:1090-103.

42. Kyte J, Doolittle RF. A simple method for displaying the hydropathic character of a protein. J Mol Biol. 1982;157:105-32.

43. Thompson JD, Higgins DG, Gibson TJ. CLUSTAL W: improving the sensitivity of progressive multiple sequence alignment through sequence weighting, position-specific gap penalties and weight matrix choice. Nucleic Acids Res. 1994;22:4673-80.

44. Tamura K, Nei M. Estimation of the number of nucleotide substitutions in the control region of mitochondrial DNA in humans and chimpanzees. Mol Biol Evol. 1993;10:512-26.

45. Drummond AJ, Suchard MA, Xie D, Rambaut A. Bayesian phylogenetics with BEAUti and the BEAST 1.7. Mol Biol Evol. 2012;29:1969-73.

46. Steel $\mathrm{M}$, Mckenzie A. Properties of phylogenetic trees generated by yuletype speciation models. Math Biosci. 2001;170:91-112.

47. Drummond AJ, Nicholls GK, Rodrigo AG, Solomon W. Estimating mutation parameters, population history and genealogy simultaneously from temporally spaced sequence data. Genetics. 2002;161:1307-20.

48. Bouckaert RR. DensiTree: making sense of sets of phylogenetic trees. Bioinformatics. 2010:26:1372-3.

49. Rambaut A, Drummond a. Tree annotator version 1.4 part of the BEAST package. 2002;

50. Rambaut A. FigTree, a graphical viewer of phylogenetic trees 2007;11:7.

51. Krogh A, Larsson B, von Heijne G, Sonnhammer EL. Predicting transmembrane protein topology with a hidden markov model: application to complete genomes. J Mol Biol. 2001;305:567-80.

52. Maddison W, Maddison D. Mesquite: a modular system for evolutionary analysis Version 3.2. 2017. https://mesquiteproject.wikispaces.com. Accessed 20 Aug 2017

53. Boore JL. Animal mitochondrial genomes. Nucleic Acids Res. 1999;27: $1767-80$.

54. Soroka M, Burzyński A. Complete female mitochondrial genome of Anodonta anatina (Mollusca: Unionidae): confirmation of a novel proteincoding gene (F-ORF). Mitochondrial DNA. 2015;26:267-9.

55. Serb JM, Lydread C. Complete mtDNA sequence of the north American freshwater mussel, Lampsilis ornata (Unionidae): an examination of the evolution and phylogenetic utility of mitochondrial genome organization in Bivalvia (Mollusca). Mol Biol Evol. 2003;20:1854-66.

56. Breton S, Burger G, Stewart DT, Blier PU. Comparative analysis of genderassociated complete mitochondrial genomes in marine mussels (Mytilus spp.). Genetics. 2006;172:1107-19.

57. Veinot, S. The mitochondrial H-ORF of the swan mussel Anodonta cygnea: analysing the expression of a unique genetic region. Acadia University, Honours Thesis. 2017. http://scholar.acadiau.ca/islandora/object/theses:2086. Accessed 10 Jul 2017.

58. Wheeler DL, Barrett T, Benson DA, Bryant SH, Canese K, Chetvernin V, et al. Database resources of the National Center for biotechnology information. Nucleic Acids Res. 2007:36:D13-21.

59. Soroka M, Burzyński A. Complete male mitochondrial genome of Anodonta anatina (Mollusca: Unionidae). Mitochondrial DNA Part A. 2016:27:1679-80.

60. Wen HB, Cao ZM, Hua D, Xu P, Ma XY, Jin W, Yuan XH, Gu RB. The complete maternally and paternally inherited mitochondrial genomes of a freshwater mussel Potamilus alatus (Bivalvia: Unionidae). PLoS One. 2017;12: e0169749.

61. Huang X-C, Rong J, Liu Y, Zhang M-H, Wan Y, Ouyang S, et al. The complete maternally and paternally inherited mitochondrial genomes of the endangered freshwater mussel Solenaia carinatus (Bivalvia: Unionidae) and implications for Unionidae taxonomy. PLoS One. 2013;8:e84352.

62. Soroka M. Characteristics of mitochondrial DNA of unionid bivalves (Mollusca: Bivalvia: Unionidae). II. Comparison of complete sequences of maternally inherited mitochondrial genomes of Sinanodonta woodiana and Unio pictorum. Folia Malacol. 2010;18:189-209.

63. McClellan DA, Palfreyman EJ, Smith MJ, Moss JL, Christensen RG, Sailsbery JK. Physicochemical evolution and molecular adaptation of the cetacean and artiodactyl cytochrome b proteins. Mol Biol Evol. 2005;22:437-55.

64. Kapp K, Schrempf S, Lemberg MK, Dobberstein B. Post-targeting functions of signal peptides. Landes. Bioscience. 2013; https:/www.ncbi.nlm.nih.gov/ books/NBK6322/. Accessed 8 Aug 2017

65. Martoglio B, Dobberstein B. Signal sequences: more than just greasy peptides. Trends Cell Biol. 1998;8:410-5. 
66. Martoglio B. Intramembrane proteolysis and post-targeting functions of signal peptides. Biochem Soc Trans. 2003;31:1243-7.

67. Hegde RS, Bernstein HD. The surprising complexity of signal sequences. Trends Biochem Sci. 2006;31:563-71.

68. Gierasch LM. Signal sequences. Biochemistry. 1989;28:923-30.

69. Roy P, Chatellard C, Lemay G, Crine P, Boileau G. Transformation of the signal peptide/membrane anchor domain of a type II transmembrane protein into a cleavable signal peptide. J Biol Chem. 1993;268:2699-704.

70. Chen $\mathrm{H}$, Kendall DA. Artificial transmembrane segments: requirements for stop transfer and polypeptide orientation. J Biol Chem. 1995;270:14115-22.

71. Milani L, Ghiselli F, Guerra D, Breton S, Passamonti M. A comparative analysis of mitochondrial ORFans: new clues on their origin and role in species with doubly uniparental inheritance of mitochondria. Genome Biol Evol. 2013;5:1408-34.

72. Bairoch A, Apweiler R. The SWISS-PROT protein sequence database and its supplement TrEMBL in 2000. Nucleic Acids Res. 2000;28:45-8.

73. Shen H-B, Yang J, Chou K-C. Euk-PLoc: an ensemble classifier for largescale eukaryotic protein subcellular location prediction. Amino Acids. 2007;33:57-67.

74. Lopes-Lima M, Froufe E, Do VT, Ghamizi M, Mock KE, Kebapçı Ü, et al. Phylogeny of the most species-rich freshwater bivalve family (Bivalvia: Unionida: Unionidae): defining modern subfamilies and tribes. Mol Phylogenet Evol. 2017;106:174-91.

75. Kyriakou E, Kravariti L, Vasilopoulos T, Zouros E, Rodakis GC. A protein binding site in the $\mathrm{M}$ mitochondrial genome of Mytilus galloprovincialis may be responsible for its paternal transmission. Gene. 2015:562:83-94.

76. Hoeh WR, Stewart DT, Sutherland BW, Zouros E. Cytochrome c oxidase sequence comparisons suggest an unusually high rate of mitochondrial DNA evolution in Mytilus (Mollusca: Bivalvia). Mol Biol Evol. 1996;13:418-21.

77. Stewart DT, Kenchington ER, Singh RK, Zouros E. Degree of selective constraint as an explanation of the different rates of evolution of genderspecific mitochondrial DNA lineages in the mussel Mytilus. Genetics. 1996; 143:1349-57.

78. Hoeh WR, Stewart DT, Guttman SI. High fidelity of mitochondrial genome transmission under the doubly uniparental mode of inheritance in freshwater mussels (Bivalvia: Unionoidea). Evolution. 2002;56:2252-61.

79. Fonseca MM, Lopes-Lima M, Eackles MS, King TL, Froufe E. The female and male mitochondrial genomes of Unio delphinus and the phylogeny of freshwater mussels (Bivalvia: Unionida). Mitochondrial DNA Part B. 2016;1: 954-7.

80. Soroka M, Burzyński A. Doubly uniparental inheritance and highly divergent mitochondrial genomes of the freshwater mussel Unio tumidus (Bivalvia: Unionidae). Hydrobiologia. 2017;(1)

81. Campbell D, Lydeard C. The Genera of Pleurobemini (Bivalvia: Unionidae: Ambleminae). 2012;30:19-38.

82. Campbell DC, Serb JM, Buhay JE, Roe KJ, Minton RL, Lydeard C. Phylogeny of north American amblemines (Bivalvia, Unionoida): prodigious polyphyly proves pervasive across genera: phylogeny of north American amblemines. Invert Biol. 2005;124:131-64.

83. Pfeiffer III J, Johnson N, Randklev C, G Howells R, Williams J. Generic reclassification and species boundaries in the rediscovered freshwater mussel "Quadrula" mitchelli (Simpson in Dall, 1896). Conserv Genet 2016;17: 279-292.

84. Araujo R, Buckley D, Nagel K-O, Machordom A. Potomida littoralis (Bivalvia, Unionidae) evolutionary history: slow evolution or recent speciation? Zool J Linn Soc. 2016. doi.wiley.com/10.1111/zoj.12470.

85. Zanatta DT, Harris AT. Phylogeography and genetic variability of the freshwater mussels (Bivalvia: Unionidae) ellipse, Venustaconcha ellipsiformis (Conrad 1836), and bleeding tooth, V. Pleasii (marsh 1891). Am Malacol Bull. 2013;31:267-79

\section{Submit your next manuscript to BioMed Central and we will help you at every step:}

- We accept pre-submission inquiries

- Our selector tool helps you to find the most relevant journal

- We provide round the clock customer support

- Convenient online submission

- Thorough peer review

- Inclusion in PubMed and all major indexing services

- Maximum visibility for your research

Submit your manuscript at www.biomedcentral.com/submit

) Biomed Central 